\title{
Congreso de la Confederación Latinoamericana de Sociedades de Anestesiología CLASA 2019
}

\author{
Congress of the Latin American Confederation of Societies \\ of Anesthesiology CLASA 2019 Why is it important to attend \\ the Congress of CLASA Argentina A19?
}

Ignacio Varela Maldonado

\section{¿Porque es importante asistir al Congreso de CLASA Argentina A19?}

D

a Confederación Latinoamericana de Sociedades de Anestesia CLASA es la Sociedad gremial que une a las Sociedades Médicas de 21 países.

Durante el desarrollo del II Congreso Argentino de Anestesiología realizado en Buenos Aires en octubre de 1949, el Argentino Dr. José César Delorme al comprobar la presencia de colegas de diversos países latinoamericanos, sugirió que el congreso podría convertirse en el Primer Congreso Latinoamericano de Anestesiología, lo que se aprobó por unanimidad por los representantes de las Sociedades Latinoamericanas presentes, las cuales fueron ocho: Argentina, Brasil, Colombia, Cuba, Chile, México, Uruguay y Venezuela.

En el libro de actas de la Asociación Argentina de Anestesiología se escribió: "En la ciudad de Buenos Aires, a los veinte y dos días del mes de octubre de mil novecientos cuarenta y nueve, reunidos los representantes de las Repúblicas Argentina Doctor José César Delorme, de los Estados Unidos de Brasil Doctor Oscar Vasconcellos Ribeiro, de Colombia Doctor Juan Marín, de Cuba Doctor Ricardo Méndez Peñate, de Chile Doctor Ernesto Frías Meneses, de México Doctor Benjamín Bandera, del Uruguay Doctor Dardo E. Vega y de Venezuela Doctor Roberto J. Lucca Escobar,

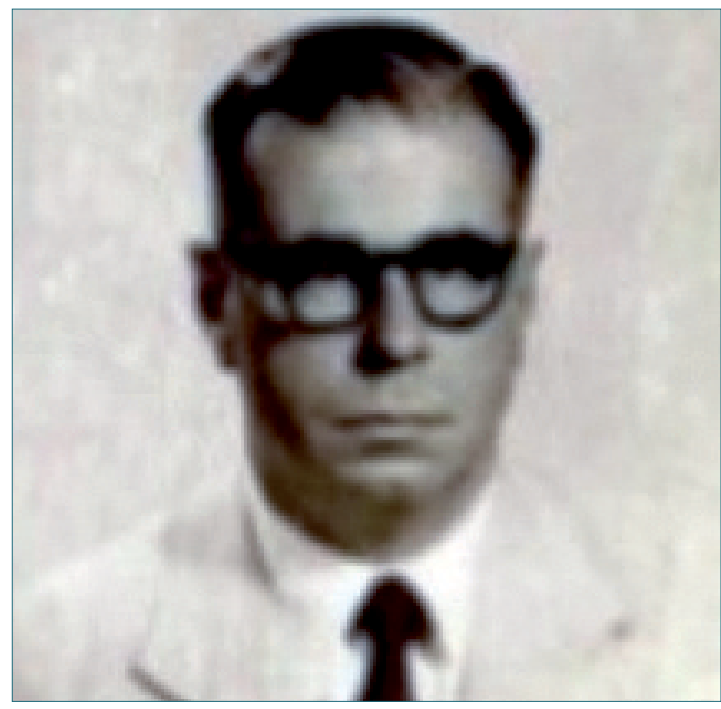

Figura 1. Dr. José Delorme.

con el objeto de difundir la anestesiología y afianzar vínculos de confraternidad resuelven la realización de los Congresos Latinoamericanos de Anestesiología que se celebrarán cada dos años". Con posterioridad adhirieron las Sociedades de Anestesiología de Paraguay, Ecuador y Perú.

Presidente de la Confederación Latinoamericana de Sociedades de Anestesiología CLASA 2018 - 2019.

Fecha de recepción: 14 de marzo de 2019

Fecha de aceptación: 14 de marzo de 2019

\section{ORCID}

https://orcid.org/0000-0012-1435

Correspondencia:

Ignacio Varela Maldonado

Email: varelami@hotmail.com 


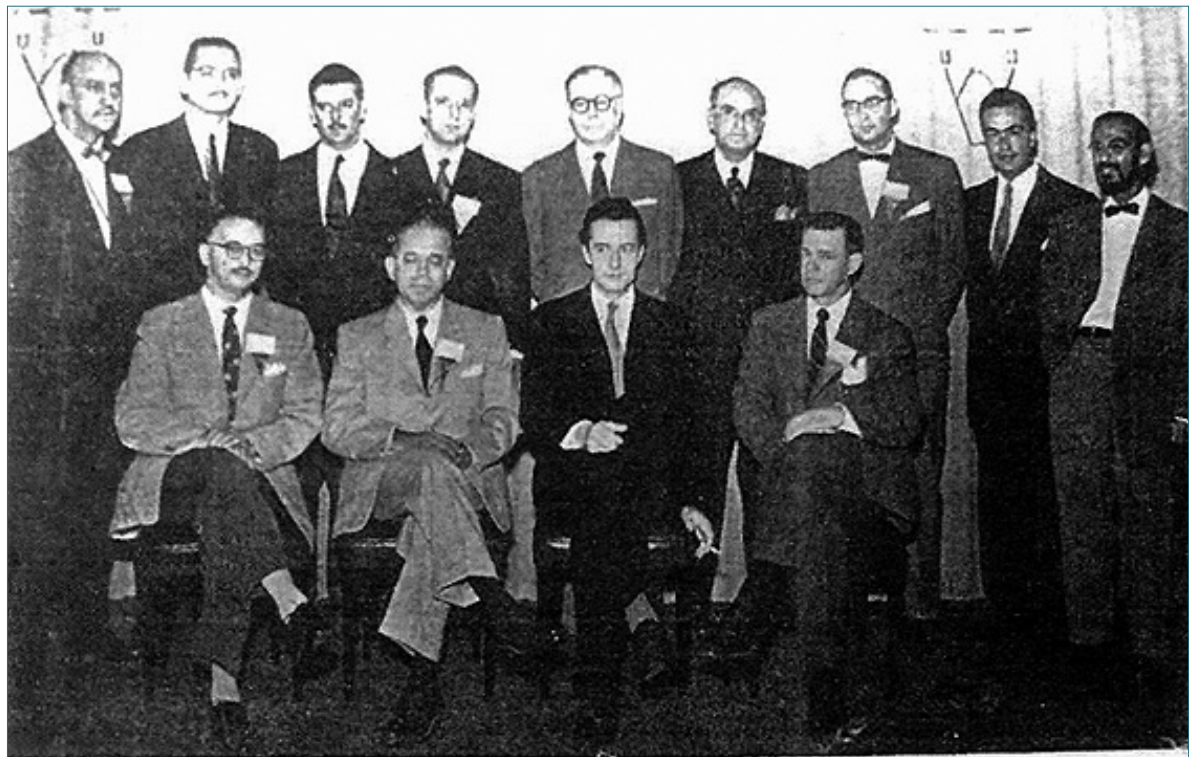

Representontes Ofieioles o Invitados de Honor ante el 20 Congreso Latinoamericano de Anestésiología.

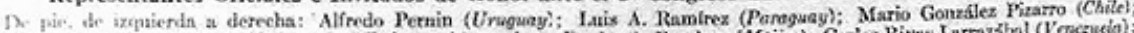

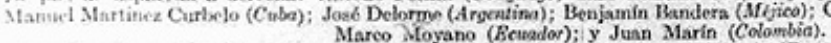
Sentadvo: Harry M. Slater (Canodía); Stevens Martin (U. S, A.) Henry Laborit (Prancia) y Vincent Collins (U.S. A.)
Figura 2. Congreso CLASA en Brasil, Sao Paulo, Brasil, septiembre de 1954, Segundo Congreso Latinoamericano de Anestesiología y primero brasileño.
El 22 de octubre de 1962, durante el desarrollo del VI Congreso Latinoamericano de Anestesiología realizado en la ciudad de Lima, Perú, se fundó la Confederación Latinoamericana de Sociedades de Anestesiología (CLASA). Esta creación fue el resultado final de los esfuerzos realizados por el anestesiólogo argentino Dr. José César Delorme. Los países fundadores fueron diez: Argentina, Bolivia, Brasil, Colombia, Chile, Ecuador, México, Perú, Uruguay y Venezuela.

Este año, del 21 al 25 de septiembre se realizará en la hermosa ciudad de Buenos Aíres Argentina el XXXV Congreso Latinoamericano organizado por la Confederación Latinoamericana de Anestesiología denominado Argentina A19 con el lema "APRENDER DESDE EL HACER".

De esta forma, Argentina ha tenido la oportunidad de organizar ya tres congresos latinoamericanos: 1949, 1967 y 1989, por lo que este año por cuarta ocasión tendrá la oportunidad de realizarlo.

La realización de los congresos latinoamericanos ha sido desde su inicio una brillante y bella oportunidad de transmitir y compartir los conocimientos científicos a las sociedades integrantes de CLASA del desarrollo de la Anestesiología que día a día se afianza, se desarrolla y se imparte con una cada vez mayor responsabilidad y ética.

Mas así mismo han permitido el que se tenga y sean aprovechados como una gran y magnífica oportunidad de nacimiento de amistades y de estrechar más las ya existentes.

Desde su inicio se tuvo la idea de que la organización fuera cada dos años y que se fuera rotando conforme al abecedario, para que por esta forma todas las sociedades integrantes de CLASA tuvieran la oportunidad de organizarlo y, así mismo, todos tuviéramos la oportunidad de conocer al país que le tocara organizarlo.

Después de ya llevar organizados, a través de 70 años 34 congresos, se puede decir con orgullo "Misión cumplida".

Nos toca a los presentes en estos tiempos continuar y engrandecer los sueños de quienes nos antecedieron y crearon lo que hoy es una realidad.

La educación médica continua constituye la mejor forma de mantener y elevar los conocimientos de una especialidad como lo es en la actualidad la Anestesiología, en la cual se tienen altos índices de nivel de desarrollo basado en conocimientos, equipo tecnológico, así como en la necesidad de que ese nivel de conocimientos requiere que el médico anestesiólogo, para mantenerse actualizado en ese desarrollo, debe asistir y/o tener acceso a los medios de comunicación actual por medio de los cuales obtendrá y tendrá acceso a ese conocimiento deseado. 


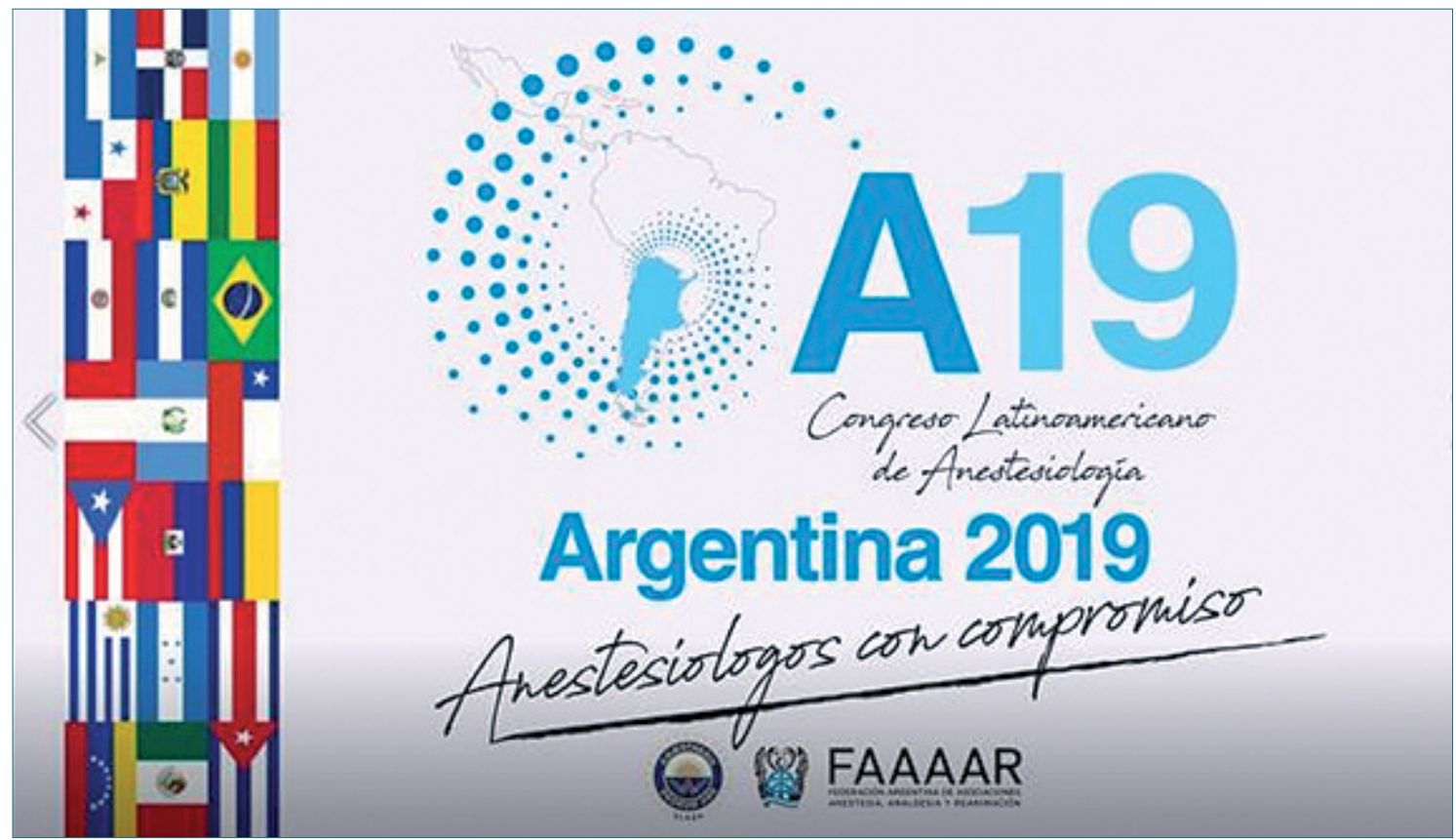

Figura 3. A19 Congreso CLASA en Argentina Buenos Aires 21-24 de septiembre de 2019.

El que el médico anestesiólogo esté actualizado constituye para él una obligación que debe de mantener, pues de esa forma responderá a las necesidades requeridas de su paciente, de su hospital.

\section{El Dr. Ignacio Chávez fundador del Instituto Nacional} de Cardiología de México, Expreso:

Un deber fundamental de todo médico es estudiar y saber; saber adquirir conocimiento, para actuar el día de mañana en beneficio de los enfermos, y esto se puede resumir señalando la imperiosa necesidad de que el médico sea un científico actualizado, es decir, en estado constante de renovación y constatación de la vigencia de sus conocimientos. Esto constituye "el culto del saber", motor intemporal que subyace a la existencia como tal de un médico que se precie de serlo.

\section{Si queremos saber, no debemos comenzar por ignorar}

CLASA, como parte de sus obligaciones, ha trabajado y debe hacerlo en una forma responsable por lograr que el médico anestesiólogo esté actualizado, y en el que la Anestesiología latinoamericana responda ante las exigencias de nuestra sociedad, y en el que la permanente educación médica continua dé garantía de que el acto anestésico es efectuado en forma responsable y con ética.
Igualmente el maestro Ignacio Chávez nos dice:

No es sabio el que sabe, sino el que recorre, porque los conoce, los caminos del saber. Aquel que no está preparado para para renovar su bagaje estará condenado a quedar atrás en el curso de un poco tiempo.

\section{El médico ayuda con lo que sabe, no con lo que ignora}

El médico debe estudiar, si la curiosidad científica no basta para dictarle esa conducta, la ética profesional le exige ofrecer al enfermo una ayuda eficaz, no simulada. Quien por no estar enterado de los avances deja sin protección a su enfermo, comete en él un fraude que la ética profesional condena.

Por ello decimos que no hay mayor forma democrática y limpia de progreso de un pueblo que la enseñanza y por ella hay que estudiar.

Es nuestro deber, como miembros de esta sociedad actual, el participar en las actividades de nuestras organizaciones. El que lo hagamos constituye la mejor forma de cumplir nuestra obligación con ética y responsabilidad. CLASA es nuestra, nosotros a través de nuestros antecesores la creamos, entre todos la mantenemos viva, pujante y actuando; entre todos le ayudamos a cumplir sus cometidos, entre todos lograremos el que nuestros pacientes tengan acceso a un mejor tratamiento médico-quirúrgico-anestésico que le dé la posibilidad de un mejor estadio de vida. 
He tenido la oportunidad de formar parte del comité organizador del XXIX Congreso realizado en Cancún México del 21 al 24 de noviembre de 2007 y estar presente en los Congresos CLASA de 2013 en Asunción Paraguay, Lima Perú en 2015 y Punta del Este, Uruguay en 2017, y puedo decir con orgullo que siempre he observado el entusiasmo, la alegría, la gran satisfacción entre los asistentes por compartir conocimientos, por conocer diversas costumbres de nuestros pueblos, por conocer la diversidad en el pensamiento y en las acciones, ver la satisfacción de participar y dar a conocer el folclor de nuestros pueblos.

La Federación Argentina de Asociaciones de Anestesia, Analgesia y Reanimación, está realizando con gran entusiasmo, con la mayor responsabilidad, con gran dedicación, y el mayor esfuerzo porque este próximo Congreso de CLASA se constituya en un gran éxito y siempre con el ideal y el pensamiento que se honre la memoria de su paisano el Dr. José César Delorme, y así mismo, en el que la unidad y amistad y el deseo de lograr que de esta unidad se refrende el pensamiento de que el acto anestésico debe ser respetado íntegramente y que la "Anestesia debe ser aplicada por anestesiólogos". Y que si mantenemos ese pensamiento con acciones, con hechos en los que mostremos que el médico anestesiólogo es capaz, es educado, es ético, es responsable y que tiene la capacidad de mostrar que la anestesia no es solo "dormir al paciente", sino que por el contrario, contribuye en grado sumo en mantener a su paciente en condiciones de estabilización fisiológica que le permita ser sometido a la cirugía programada.

Así mismo, hemos observado con gran satisfacción el que la Sociedad Chilena de Anestesia ha decidido y ha mostrado en un ambiente de que la ennoblece en grado máximo en unirse y contribuir con su presencia física y en unidad gremial en la organización y en la asistencia al próximo Congreso.

El Comité Ejecutivo de CLASA envía a la Sociedad Chilena su sincero reconocimiento por este gran gesto de entrega decidida y eficaz y por su cooperación para que este Congreso de la Anestesiología Latinoamericana se constituya en una magnífica oportunidad de estrechar la unidad y amistad entre su pueblo vecino y aún más allá.

Lo anterior lo celebro y seguro estoy de que ese acto será un parteaguas para el futuro de nuestra querida Confederación y que contribuirá mucho a que otras sociedades igualmente decidan participar y contribuir a que el Congreso sea un motivo de mayor acercamiento y contribuya al desarrollo de todas las sociedades miembros.

Así mismo, y dentro del propio programa acadé- mico, se llevará a cabo el cuarto encuentro internacional de residentes lo cual ocasionara el encuentro de quienes en el futuro serán los anestesiólogos y en quienes se depositara la confianza y el conocimiento para que sean ellos quienes conduzcan posteriormente a nuestras instituciones y sean los herederos del conocimiento y de los nobles sentimientos de quienes fundaron nuestra amada institución.

Les invito a todos a participar y que estemos todos seguros que nuestra, porque repito es de todos nosotros la Confederación Latinoamericana de Sociedades de Anestesiología, es un gran relicario de nuestros sueños, de nuestros anhelos y de nuestros grandes y bellos deseos de contribuir en lo que respecta a nuestra bella y dulce especialidad médica a que siga contribuyendo al desarrollo de nuestros pueblos.

Y como lo indican nuestros estatutos, el día 21 tendremos la asamblea ordinaria de delegados de CLASA destacando en que en ella se nombrara al nuevo comité ejecutivo que iniciará sus actividades oficialmente el 1 de enero de 2020.

El programa académico que se ha programado incluye cursos precongreso, talleres, eventos de simulación y conferencias con profesores de muy grande nivel académico miembros de las diversas sociedades integrantes de CLASA como de algunos otros países, quienes con sus ponencias y su espíritu de compartir sus conocimientos hacen que el programa académico destaque grandemente.

Así mismo, y como parte del programa, tendremos eventos de carácter social dignos del propio Congreso, con grandes sorpresas que nos permitirán dentro de la alegría propia de nuestros pueblos convivir y compartir nuestra amistad.

Destacará dentro de estas actividades la tradicional noche latinoamericana que será una gran oportunidad de manifestar las tradiciones de cada una de las naciones.

Se tendrán otros eventos que serán la gran sorpresa de este bello y gran Congreso.

Por todo lo anterior y para que lo nuestro, para lo que forjaron nuestros antecesores y para lo que heredaremos es por lo que debemos asistir y hacernos presentes en este ya muy próximo XXXV Congreso Latinoamericano de Anestesiología Argentina A19. Los esperamos a todos ustedes con gran alegría, y el comité ejecutivo de CLASA 2018 - 2019 les recibirá con todo nuestro afecto.

Con todo afecto

Dr. Ignacio Varela Maldonado Presidente CLASA 2018 - 2019 